\title{
Sociodemographic Factors and Denture Satisfaction
}

\author{
${ }^{1}$ Balendra Pratap Singh, ${ }^{2}$ Kedar Nath Pradhan, ${ }^{3}$ Reema Tua, ${ }^{4}$ Arvind Tripathi \\ ${ }^{1}$ Assistant Professor, Department of Prosthodontics, CSM Medical University, Lucknow, Uttar Pradesh, India \\ ${ }^{2}$ Deputy Director, Department of Dentistry, Gangtok Hospital, Gangtok, Sikkim, India \\ ${ }^{3}$ Associate Professor, Department of Social and Preventive Medicine, CSM Medical University, Lucknow, Uttar Pradesh, India \\ ${ }^{4}$ Professor, Department of Prosthodontics, Saraswati Dental College, Lucknow, Uttar Pradesh, India
}

Correspondence: Balendra Pratap Singh, Assistant Professor, Department of Prosthodontics, Flat No. 101, New Teacher's Apartment, TG Hostel Chowk, Lucknow-226003, Uttar Pradesh, India, Phone: +91-09839121151, e-mail: balendra02@yahoo.com

\section{ABSTRACT}

Objective: Evaluation of the quality of satisfaction toward denture treatment.

Background: Various articles suggested denture satisfaction in view of dentist but data on the patient satisfaction to denture treatment on the basis of clinical variables were contradictory. This showed that other factors like sociodemographic variables may affect patient satisfaction.

Materials and methods: A total of 100 subjects (filling inclusion criteria) who were using denture for at least 2 months were used as the sample size of this study $(n=100)$ and were divided into five groups on the basis of sociodemographic variables. Questionnaires consisting of 38 questions (positive and negative attitude toward denture satisfaction) related to patients' perception of clinical outcome in different domains were scored by the subjects. Questions reflecting positive attitude were scored as 2, 1 or 0 (yes, uncertain and no respectively) and reversely for the negative questions. Statistical tests include Student t-test and $p$-value (for level of significance) $(p<0.05)$.

Results: There was a significant difference in denture satisfaction related to comfort, health and denture care between age subgroups (45-65 years and above 65 years); the level of satisfaction was not significantly different between genders.

Conclusion: Patients' sociodemographic variables were influential factors on denture satisfaction.

Keywords: Dentures, Satisfaction, Compliance, Socioeconomic status, Educational status.

\section{INTRODUCTION}

Although the prevalence of edentulism is falling in all age groups, ${ }^{1}$ the number of older people is still increasing. India being a developing country and having a huge population, lacks awareness and management about edentulous state. ${ }^{2,3}$ Rehabilitating edentulous patients with complete dentures has been the treatment of choice. One study demonstrated a poor correlation between patient satisfaction and clinical variables ${ }^{4}$ and that clinicians' assessment of the quality of denturesupporting tissues are poor predictors of patients' satisfaction. ${ }^{5}$ One primary goal in therapies for chronic conditions such as edentulism is improvement in function rather than cure, and therefore it is patient-based outcomes that are most important. ${ }^{6}$ Studies in edentulous subjects strongly support the concept that patient-based measures are more sensitive than functional measures for detecting difference between treatments. ${ }^{7-9}$ Patients' perceptions about subjective experience of their denture are also important for dentists to motivate complete denture wearers.

The successes of complete dentures are dependent on the multiple factors: ${ }^{10}$ patient perception is important for successful treatment. The author of the present study hypothesized that apart from biological and technical considerations in fabrication of complete dentures ${ }^{11,12}$ different parameters such as age, gender, literacy level, socioeconomic and marital status may affect satisfaction toward complete dentures. To evaluate this, a standardized questionnaire that included domains, such as mastication, appearance, speech, comfort, health, denture care and social status was used to determine satisfaction levels with different parameters.

\section{MATERIALS AND METHODS}

Subjects were selected from Prosthodontic Clinic of CSM Medical University, Lucknow. The subjects who fulfilled the following inclusion criteria were considered for the study: Those who were free from systemic diseases (on the basis of medical history and physician consultation) which affected oral functions, first time denture wearer, period of edentulousness between 6 months to 1 year and class 1 completely edentulous patient. ${ }^{13}$ Patients were excluded if they belonged to class 2,3 or 4 types, single complete dentures and temporomandibular joint disorders. This study was approved by the institutional ethical committee. The age of the patients ranged from 45 to 79 years. Informed consent was obtained regarding their voluntary participation in the study. Participants were asked about their sociodemographic characteristics including age, gender, literacy, socioeconomic and marital status. The socioeconomic status was divided into upper high, upper middle and poor class based on the monthly income of the subjects. ${ }^{14}$ 
In this study, a total number of 136 subjects were enrolled that had fulfilled the inclusion criteria and had received complete dentures. All the included subjects were divided into five groups on the basis of age, gender, literacy (illiterate subjects were questioned orally by a lay person and ticked choice on behalf of subjects), socioeconomic and marital status:

\section{Group A: Age}

Subgroup $1-45$ to 65 years

Subgroup 2-above 65 years

Group B: Gender

Subgroup 1-male

Subgroup 2-female

Group C: Literacy level

Subgroup 1-illiterate

Subgroup 2-primary level

Subgroup 3-secondary level

Subgroup 4-graduate level

Subgroup 5-postgraduate level

Group D: Socioeconomic status

Subgroup 1-upper high

Subgroup 2-upper middle

Subgroup 3-poor

Group E: Marital status

Subgroup 1-married

Subgroup 2-widow/widower.

Denture therapy was completed by postgraduate student dentists with four years clinical experience under the direction of faculty in the department of prosthodontics using standard clinical and laboratory procedures. Their quality was assessed on the basis of quantifying method for denture quality by Sato et $\mathrm{al}^{15}$ as per which only good quality dentures were selected for the study. Before starting denture therapy, treatment eagerness (own will or advised by others) and priority for denture treatment esthetics, phonetics or function were questioned. At the denture insertion appointment, printed and verbal postinsertion instructions were given to each patient with particular emphasis to report to the prosthodontic clinic after 2 months for follow-up. Those patients who had regularly (every day for at least 8 hours) using their dentures were included in the study. A standardized questionnaire of 38 questions was given. Particular questions were related to either positive or negative attitudes. This questionnaire had 20 and 18 number of positive and negative attitudes questions. The questionnaire had domains of mastication, appearance, speech, comfort, health, denture care and social status. Questions (1-38) were recorded as yes, no or uncertain. All the items of positive nature were scored as 2, 1 or 0 (yes, uncertain and no respectively) and reversely for the negative items.

Data analysis was carried out using statistical package for Social Sciences (version 15.0) (SPSS Inc, Chicago, IL, USA). Significance of percentage error of two groups was tested by
Student t-test and for level of significance p-value was used. A probability value of less than 0.05 was considered statistically significant.

\section{RESULTS}

The present study was conducted on 100 subjects who had received their complete dentures and were administered with questionnaires containing questions related to quality of satisfaction toward complete dentures. After completion of the questionnaires, the scoring was done as per groups on the basis of different domains.

Table 1 showed that esthetics and function were significant priority for denture treatment for subgroup 1 and subgroup 2 respectively. Level of satisfaction was higher in subgroup 2 subjects regarding their mastication, appearance, comfort, health, denture care and social status except speech in which subgroup 1 subjects showed more satisfaction (Table 2).

Priority to esthetics was significant with female and priority to function was significant with male (Table 3). Level of satisfaction was more in male with relation to mastication, appearance, speech, health and social status except comfort, denture care in which females were more satisfied (Table 4).

Level of satisfaction in mastication, appearance, speech, comfort, health, denture care and social status were found statistically significant with literacy level. Majority of illiterate patients showed partial satisfaction or dissatisfaction (Table 6).

Level of satisfaction was higher in upper high income group regarding appearance, speech, comfort, denture care and social status but significantly only in case of social status. Upper middle income group was more satisfied with mastication and significantly satisfied with health (Table 8).

The level of satisfaction was higher in married than widow/ widower patient but significantly only with comfort (Table 10).

\begin{tabular}{|c|c|c|c|c|}
\hline \multicolumn{5}{|c|}{ Treatment eagerness } \\
\hline \multirow[t]{2}{*}{$\begin{array}{l}\text { Treatment } \\
\text { eagerness }\end{array}$} & \multicolumn{2}{|c|}{$\begin{array}{c}\text { Group } 1 \\
45-65 \text { years }(n=50)\end{array}$} & \multicolumn{2}{|c|}{$\begin{array}{c}\text { Group } 2 \\
\text { Above } 65 \text { years }(n=50)\end{array}$} \\
\hline & No. & $\%$ & No. & $\%$ \\
\hline Own will & 41 & 82 & 33 & 66 \\
\hline Advised by others & 9 & 18 & 17 & 34 \\
\hline \multicolumn{5}{|c|}{$\chi^{2}=3.33, p=0.06$} \\
\hline \multicolumn{5}{|c|}{ Priority for denture treatment } \\
\hline \multirow[t]{2}{*}{ Priority } & \multicolumn{2}{|c|}{ Group 1} & \multicolumn{2}{|c|}{ Group 2} \\
\hline & No. & $\%$ & No. & $\%$ \\
\hline Good looks & 38 & 76 & 14 & 28 \\
\hline Eating & 12 & 24 & 36 & 72 \\
\hline Speech & 0 & 0 & 0 & 0 \\
\hline \multicolumn{5}{|c|}{$\chi^{2}=23.08, p<0.001$} \\
\hline
\end{tabular}


Table 2: Age-wise distribution of patients regarding quality of satisfaction toward complete denture

\begin{tabular}{|c|c|c|c|c|c|c|}
\hline \multirow[t]{2}{*}{ Satisfaction } & & \multicolumn{2}{|c|}{$\begin{array}{c}\text { Subgroup } 1 \\
\text { 45-65 years } \\
\quad(n=50)\end{array}$} & \multicolumn{2}{|c|}{$\begin{array}{l}\text { Subgroup } 2 \\
\text { Above } 65 \text { years } \\
\quad(n=50)\end{array}$} & \\
\hline & & No. & $\%$ & No. & $\%$ & \\
\hline \multirow[t]{3}{*}{ Mastication } & a. Satisfactory & 24 & 48 & 25 & 50 & Total no. of patients \\
\hline & b. Partially satisfactory & 14 & 28 & 13 & 26 & Subgroup $1-50$, Subgroup $2-50$ \\
\hline & c. Not satisfactory & 11 & 22 & 13 & 26 & $\chi^{2}=0.22, p=0.89$ \\
\hline \multirow[t]{3}{*}{ Appearance } & a. Satisfactory & 3 & 26 & 17 & 34 & Total no. of patients \\
\hline & b. Partially satisfactory & 10 & 20 & 6 & 12 & Subgroup $1-50$, Subgroup $2-50$ \\
\hline & c. Not satisfactory & 27 & 54 & 27 & 54 & $\chi^{2}=1.53, p=0.46$ \\
\hline \multirow[t]{3}{*}{ Speech } & a. Satisfactory & 18 & 36 & 16 & 32 & Total no. of patients \\
\hline & b. Partially satisfactory & 10 & 20 & 12 & 24 & Subgroup $1-50$, Subgroup $2-50$ \\
\hline & c. Not satisfactory & 22 & 44 & 32 & 44 & $\chi^{2}=0.15, p=0.94$ \\
\hline \multirow[t]{3}{*}{ Comfort } & a. Satisfactory & 18 & 36 & 35 & 70 & Total no. of patients \\
\hline & b. Partially satisfactory & 14 & 28 & 10 & 20 & Subgroup $1-50$, Subgroup $2-50$ \\
\hline & c. Not satisfactory & 18 & 36 & 5 & 10 & $\chi^{2}=13.47, p<0.001$ \\
\hline \multirow[t]{3}{*}{ Health } & a. Satisfactory & 12 & 24 & 23 & 46 & Total no. of patients \\
\hline & b. Partially satisfactory & 13 & 26 & 10 & 20 & Subgroup $1-50$, Subgroup $2-50$ \\
\hline & c. Not satisfactory & 25 & 50 & 17 & 34 & $\chi^{2}=5.37, p=0.07$ \\
\hline \multirow[t]{3}{*}{ Denture care } & a. Satisfactory & 36 & 72 & 42 & 84 & Total no. of patients \\
\hline & b. Partially satisfactory & 5 & 10 & 3 & 6 & Subgroup $1-50$, Subgroup $2-50$ \\
\hline & c. Not satisfactory & 9 & 18 & 5 & 10 & $\chi^{2}=2.10, p=0.35$ \\
\hline \multirow[t]{3}{*}{ Social status } & a. Satisfactory & 23 & 46 & 25 & 50 & Total no. of patients \\
\hline & b. Partially satisfactory & 16 & 32 & 14 & 28 & Subgroup $1-50$, Subgroup $2-50$ \\
\hline & c. Not satisfactory & 11 & 22 & 11 & 22 & $\chi^{2}=0.22, p=0.90$ \\
\hline
\end{tabular}

\begin{tabular}{|c|c|c|c|c|}
\hline \multicolumn{5}{|c|}{ Treatment eagerness } \\
\hline \multirow{2}{*}{$\begin{array}{l}\text { Treatment } \\
\text { eagerness }\end{array}$} & \multicolumn{2}{|c|}{ Male $(n=50)$} & \multicolumn{2}{|c|}{ Female $(n=50)$} \\
\hline & No. & $\%$ & No. & $\%$ \\
\hline Own will & 61 & 76.3 & 16 & 80 \\
\hline Advised by others & 19 & 23.7 & 4 & 20 \\
\hline \multicolumn{5}{|l|}{$\chi^{2}=0.13, p=0.72$} \\
\hline \multicolumn{5}{|c|}{ Priority for denture treatment } \\
\hline \multirow[t]{2}{*}{ Priority } & \multicolumn{2}{|c|}{ Male } & \multicolumn{2}{|c|}{ Female } \\
\hline & No. & $\%$ & No. & $\%$ \\
\hline Good looks & 6 & 7.5 & 10 & 50 \\
\hline Eating & 74 & 92.5 & 10 & 50 \\
\hline Speech & 0 & 0 & 0 & 0 \\
\hline
\end{tabular}

\section{DISCUSSION}

In India, data on assessment of denture satisfaction after placement of maxillary and mandibular complete dentures is limited. Therefore, the present study was designed to assess the opinion of the prostheses worn by the subjects reporting to the Prosthodontics Department of CSM Medical University, Lucknow. This would enable subjects to indicate their satisfaction regarding the prostheses and perhaps serve as a guideline for the prosthodontists to pay increased attention to the factors of patient concern.

The present study was carried out in 100 edentulous subjects on the basis of questionnaires pertaining to level of satisfaction with complete dentures 2 months after dentures' insertion. Quality of satisfaction questionnaires includes the domains of mastication, appearance, speech, comfort, health, denture care and social status of the subjects.

Regarding eagerness of subjects toward denture treatment in age group category, it was found that maximum number of subgroup 1 subjects was self-motivated whereas subgroup 2 subjects were motivated by others for the treatment. In this finding, it is obvious that the subgroup 1 subjects at this age are socially active, professionally engaged and most of them are economically sound, for which the treatment is mandatory. Whereas in subgroup 2, age compromise, social and economic dependence and in some cases medically compromised conditions like; diabetes mellitus, hypertension, retinopathy, etc. limited for seeking denture treatment. In priority for denture treatment, subgroup 1 subjects were more conscious about their esthetics and subgroup 2 subjects were concerned toward functions. Subgroup 2 subjects consisted of potentially active 
Table 4: Gender-wise distribution of patients regarding quality of satisfaction toward complete denture

\begin{tabular}{|c|c|c|c|c|c|c|}
\hline \multirow[t]{2}{*}{ Satisfaction } & & \multicolumn{2}{|c|}{ Male $(n=80)$} & \multicolumn{2}{|c|}{ Female $(n=20)$} & \\
\hline & & No. & $\%$ & No. & $\%$ & \\
\hline \multirow[t]{3}{*}{ Mastication } & a. Satisfactory & 40 & 50 & 9 & 45 & Total no. of patients \\
\hline & b. Partially satisfactory & 20 & 25 & 5 & 25 & Male-80, Female-20 \\
\hline & c. Not satisfactory & 20 & 25 & 6 & 30 & $\chi^{2}=0.24, p=0.89$ \\
\hline \multirow[t]{3}{*}{ Appearance } & a. Satisfactory & 24 & 30 & 5 & 25 & Total no. of patients \\
\hline & b. Partially satisfactory & 13 & 16.25 & 3 & 15 & Male-80, Female-20 \\
\hline & c. Not satisfactory & 43 & 53.75 & 12 & 60 & $\chi^{2}=1.39, p=0.50$ \\
\hline \multirow[t]{3}{*}{ Speech } & a. Satisfactory & 28 & 35 & 4 & 20 & Total no. of patients \\
\hline & b. Partially satisfactory & 16 & 20 & 6 & 30 & Male-80, Female-20 \\
\hline & c. Not satisfactory & 36 & 45 & 10 & 50 & $\chi^{2}=1.94, p=0.48$ \\
\hline \multirow[t]{3}{*}{ Comfort } & a. Satisfactory & 43 & 53.75 & 10 & 50 & Total no. of patients \\
\hline & b. Partially satisfactory & 17 & 21.25 & 6 & 30 & Male-80, Female-20 \\
\hline & c. Not satisfactory & 20 & 25.00 & 4 & 20 & $\chi^{2}=0.74, p=0.60$ \\
\hline \multirow[t]{3}{*}{ Health } & a. Satisfactory & 34 & 42.50 & 3 & 15 & Total no. of patients \\
\hline & b. Partially satisfactory & 16 & 20.00 & 6 & 30 & Male-80, Female-20 \\
\hline & c. Not satisfactory & 30 & 37.50 & 11 & 55 & $\chi^{2}=5.19, p=0.07$ \\
\hline \multirow[t]{3}{*}{ Denture care } & a. Satisfactory & 55 & 69.30 & 15 & 75 & Total no. of patients \\
\hline & b. Partially satisfactory & 10 & 11.95 & 3 & 15 & Male-80, Female-20 \\
\hline & c. Not satisfactory & 15 & 18.75 & 2 & 10 & $\chi^{2}=1.39, p=0.50$ \\
\hline \multirow[t]{3}{*}{ Social status } & a. Satisfactory & 40 & 50 & 9 & 45 & Total no. of patients \\
\hline & b. Partially satisfactory & 20 & 25 & 7 & 35 & Male-80, Female-20 \\
\hline & c. Not satisfactory & 20 & 25 & 4 & 20 & $\chi^{2}=0.84, p=0.66$ \\
\hline
\end{tabular}

Table 5: Educational status-wise distribution of patients regarding their denture compliance

\begin{tabular}{|c|c|c|c|c|c|c|c|c|c|c|}
\hline \multicolumn{11}{|c|}{ Treatment eagerness } \\
\hline \multirow[t]{2}{*}{ Treatment eagerness } & \multicolumn{2}{|c|}{$\begin{array}{c}\text { Illiterate (I) } \\
\quad(n=24)\end{array}$} & \multicolumn{2}{|c|}{$\begin{array}{l}\text { Primary level } \\
(P L)(n=11)\end{array}$} & \multicolumn{2}{|c|}{$\begin{array}{l}\text { Secondary level } \\
(S L)(n=34)\end{array}$} & \multicolumn{2}{|c|}{$\begin{array}{l}\text { Graduate level } \\
(G L)(n=17)\end{array}$} & \multicolumn{2}{|c|}{$\begin{array}{c}\text { Postgraduate level } \\
\quad(P G L)(n=14)\end{array}$} \\
\hline & No. & $\%$ & No. & $\%$ & No. & $\%$ & No. & $\%$ & No. & $\%$ \\
\hline Own will & 13 & 54 & 7 & 64 & 28 & 82 & 10 & 59 & 12 & 86 \\
\hline Advised by others & 11 & 46 & 4 & 36 & 6 & 18 & 7 & 41 & 2 & 14 \\
\hline \multicolumn{11}{|l|}{$\chi^{2}=8.21, p<0.08$} \\
\hline \multicolumn{11}{|c|}{ Priority for denture treatment } \\
\hline Priority & \multicolumn{2}{|c|}{ Illiterate } & \multicolumn{2}{|c|}{ Primary level } & \multicolumn{2}{|c|}{ Secondary level } & \multicolumn{2}{|c|}{$\underline{\text { Graduate level }}$} & \multicolumn{2}{|c|}{ Postgraduate level } \\
\hline Good looks & 3 & 12.5 & 1 & 9.10 & 5 & 15 & 3 & 18 & - & - \\
\hline Eating & 21 & 87.5 & 10 & 90.9 & 29 & 85 & 14 & 82 & 14 & 100 \\
\hline Speech & 0 & 0 & 0 & 0 & 0 & 0 & 0 & 0 & 0 & 0 \\
\hline
\end{tabular}

age group which require social and professional acceptance in which esthetic concern is of utmost importance. On the other hand, the subgroup 2 conscious of retired persons, age-wise and socially compromised for which functional aspect is more concerned.

While considering the quality of satisfaction toward complete denture therapy, subgroup 2 subjects (above 65 years) were more satisfied with their dentures regarding all domains except speech where the subgroup 1 subjects (45-65 years) were more satisfied. This finding may be due to the fact that the aging population have overwhelming desire for satisfaction at all level of functions in their daily life which have been fulfilled by their dentures. Other reasons might be due to older subjects were more satisfied with poorly fitting dentures and were less prepared to take trouble to achieve denture improvement. ${ }^{16}$ The older subjects also had poorer stereognostic 
Table 7: Socioeconomic status-wise distribution of patients regarding their denture compliance

\begin{tabular}{|c|c|c|c|c|c|c|}
\hline \multicolumn{7}{|c|}{ Treatment eagerness } \\
\hline \multirow[t]{2}{*}{ Treatment eagerness } & \multicolumn{2}{|c|}{ Upper high $(n=24)$} & \multicolumn{2}{|c|}{ Upper middle $(n=26)$} & \multicolumn{2}{|c|}{ Poor $(n=50)$} \\
\hline & No. & $\%$ & No. & $\%$ & No. & $\%$ \\
\hline Own will & 21 & 87.5 & 21 & 80.8 & 35 & 70 \\
\hline Advised by others & 3 & 12.5 & 5 & 19.2 & 15 & 30 \\
\hline \multicolumn{7}{|l|}{$\chi^{2}=3.09, p=0.21$} \\
\hline \multicolumn{7}{|c|}{ Priority for denture treatment } \\
\hline Priority & \multicolumn{2}{|c|}{ Upper high $(n=24)$} & \multicolumn{2}{|c|}{ Upper middle $(n=26)$} & \multicolumn{2}{|c|}{ Poor $(n=50)$} \\
\hline Good looks & 2 & 8.40 & 4 & 15.4 & 1 & 2 \\
\hline Eating & 2 & 91.60 & 22 & 84.62 & 49 & 98 \\
\hline Speech & 0 & 0 & 0 & 0 & 0 & 0 \\
\hline
\end{tabular}

Table 8: Socioeconomic status-wise distribution of patients regarding quality of satisfaction toward complete denture

\begin{tabular}{|c|c|c|c|c|c|c|c|c|}
\hline \multirow[t]{2}{*}{ Satisfaction } & & \multicolumn{2}{|c|}{ Upper high } & \multicolumn{2}{|c|}{ Upper middle } & \multicolumn{2}{|c|}{ Poor } & \\
\hline & & No. & $\%$ & No. & $\%$ & No. & $\%$ & \\
\hline \multirow[t]{3}{*}{ Mastication } & a. Satisfactory & 12 & 50.0 & 15 & 57.7 & 22 & 44.0 & \multirow{3}{*}{$\begin{array}{l}\text { Total no. of patients } \\
\text { Upper high-24, Upper } \\
\text { middle-26, Poor-50 } \\
\chi^{2}=7.12, p=0.13\end{array}$} \\
\hline & b. Partially satisfactory & 10 & 41.7 & 5 & 19.2 & 12 & 24.0 & \\
\hline & c. Not satisfactory & 2 & 08.3 & 6 & 23.1 & 16 & 32.0 & \\
\hline \multirow{5}{*}{ Appearance } & & & & & & & & \multirow{5}{*}{$\begin{array}{l}\text { Total no. of patients } \\
\text { Upper high-24, Upper } \\
\text { middle-26, Poor-50 } \\
\chi^{2}=12.26, \mathrm{p}=0.016\end{array}$} \\
\hline & a. Satisfactory & 13 & 54.2 & 9 & 34.6 & 8 & 16.0 & \\
\hline & b. Partially satisfactory & 2 & 08.3 & 3 & 11.5 & 11 & 22.0 & \\
\hline & c. Not satisfactory & 9 & 37.5 & 14 & 53.8 & 31 & 62.0 & \\
\hline & & & & & & & & \\
\hline \multirow[t]{4}{*}{ Speech } & a. Satisfactory & 11 & 45.8 & 9 & 34.6 & 13 & 26.0 & \multirow{4}{*}{$\begin{array}{l}\text { Total no. of patients } \\
\text { Upper high-24, Upper } \\
\text { middle-26, Poor-50 } \\
\chi^{2}=4.06, p=0.40\end{array}$} \\
\hline & b. Partially satisfactory & 3 & 12.5 & 7 & 26.9 & 11 & 22.0 & \\
\hline & c. Not satisfactory & 10 & 47.7 & 10 & 38.5 & 26 & 52.0 & \\
\hline & & & & & & & & \\
\hline \multirow[t]{4}{*}{ Comfort } & a. Satisfactory & 18 & 75.0 & 14 & 53.8 & 1 & 42.0 & \multirow{4}{*}{$\begin{array}{l}\text { Total no. of patients } \\
\text { Upper high-24, Upper } \\
\text { middle-26, Poor-50 } \\
\chi^{2}=7.76, p=0.101\end{array}$} \\
\hline & b. Partially satisfactory & 4 & 16.7 & 5 & 19.2 & 14 & 28.0 & \\
\hline & c. Not satisfactory & 2 & 08.3 & 7 & 26.9 & 15 & 30.0 & \\
\hline & & & & & & & & \\
\hline \multirow[t]{4}{*}{ Health } & a. Satisfactory & 12 & 50.0 & 14 & 53.8 & 11 & 22.0 & \multirow{4}{*}{$\begin{array}{l}\text { Total no. of patients } \\
\text { Upper high-24, Upper } \\
\text { middle-26, Poor-50 } \\
\chi^{2}=20.47, p=0.001\end{array}$} \\
\hline & b. Partially satisfactory & 7 & 29.2 & 8 & 30.7 & 8 & 16.0 & \\
\hline & c. Not satisfactory & 5 & 20.8 & 4 & 15.3 & 31 & 62.0 & \\
\hline & & & & & & & & \\
\hline \multirow[t]{4}{*}{ Denture care } & a. Satisfactory & 24 & 100 & 18 & 69.2 & 35 & 70.0 & \multirow{4}{*}{$\begin{array}{l}\text { Total no. of patients } \\
\text { Upper high-24, Upper } \\
\text { middle-26, Poor-50 } \\
\chi^{2}=9.49, \mathrm{p}=0.05\end{array}$} \\
\hline & b. Partially satisfactory & - & - & 3 & 11.5 & 5 & 10.0 & \\
\hline & c. Not satisfactory & - & - & 5 & 19.2 & 10 & 20.0 & \\
\hline & & & & & & & & \\
\hline \multirow[t]{3}{*}{ Social status } & a. Satisfactory & 16 & 66.7 & 12 & 46.2 & 22 & 44.0 & \multirow{3}{*}{$\begin{array}{l}\text { Total no. of patients } \\
\text { Upper high-24, Upper } \\
\text { middle-26, Poor-50 } \\
\chi^{2}=11.71, p=0.02\end{array}$} \\
\hline & b. Partially satisfactory & 8 & 33.3 & 9 & 34.6 & 11 & 22.0 & \\
\hline & c. Not satisfactory & - & - & 5 & 19.2 & 17 & 34.0 & \\
\hline
\end{tabular}

ability than the younger ones. The presence of prosthesis appeared to improve stereognostic ability. Subjects with poorer stereognostic ability appeared more satisfied with their rehabilitation than did those with better stereognostic ability. ${ }^{17}$
Regarding eagerness toward denture treatment in gender category, female were self-motivated whereas the male were advised by others for the same. This finding clearly showed that females are more aware and concerned about their denture 
Table 9: Marital status-wise distribution of patients regarding their denture compliance

Treatment eagerness

\begin{tabular}{lccccc}
\hline \multirow{2}{*}{$\begin{array}{l}\text { Treatment } \\
\text { eagerness }\end{array}$} & \multicolumn{2}{c}{ Married $(n=82)$} & & \multicolumn{3}{c}{ Widow/widower $(n=18)$} \\
\cline { 2 - 3 } Own will & No. & $\%$ & & No. & $\%$ \\
Advised by others & 13 & 15.85 & & 3 & 15 \\
\hline
\end{tabular}

$\chi^{2}=0.07, \mathrm{p}=0.78$

Priority for denture treatment

\begin{tabular}{lccccc} 
Priority & \multicolumn{2}{c}{ Married $(n=82)$} & & Widow/widower $(n=18)$ \\
\cline { 2 - 3 } \cline { 5 - 6 } Good looks & 15 & 18.3 & & 2 & 11.11 \\
Eating & 67 & 81.7 & & 16 & 88.89 \\
Speech & 0 & 0 & & 0 & 0 \\
\hline
\end{tabular}

$\chi^{2}=0.54, p=0.46$

treatment to restore their lost esthetic, social well-being and function. There were equal number of opinions of female toward function and esthetics for priority of making dentures. Whereas the male were more concerned for the function. This showed that females were equally conscious about their esthetic as well as function than males after denture treatment. $^{18}$

While considering the genders in this study, the majority of the denture wearer was males. It is because most of the Indian females are housewives. They have limited social interaction and the male/female ratio was also higher in the study population. ${ }^{19}$ The quality of satisfaction in this study has been found that male were more satisfied than female in mastication, appearance, speech and health. There was equal satisfaction level in comfort and social status of both the groups. In denture care, females were found more satisfied than males. It may appear because male patients are more complied toward denture treatment and satisfaction than their female counterparts. Taylor $^{20}$ surveyed 29 males and 30 females complete denture patients ranging from 52 to 92 years of age and found that male patients were more satisfied with dentures than female, which is similar to the present finding. Manal A Awad ${ }^{21}$ and Baer $^{22}$ also showed that patient satisfaction with conventional dental prostheses is highly dependent on gender.

The eagerness of treatment of postgraduate/professional level of literate group of subjects was found to be self-motivated followed by other literate groups. Whereas the illiterate group of subjects has to be motivated by others for the denture treatment (Table 5). This finding revealed that with the increase in literacy rate, there is increase in treatment motivations, which may be related to awareness toward denture treatment. The need for denture treatment for esthetics as well as function, which is found higher with increased literacy level. Whereas illiterate group was mainly concerned about their functions. This showed that with increase literacy rate there is awareness for esthetics as well as function toward denture treatment.

Table 10: Marital status-wise distribution of patients regarding quality of satisfaction toward complete denture

\begin{tabular}{|c|c|c|c|c|c|c|}
\hline \multirow[t]{2}{*}{ Satisfaction } & & \multicolumn{2}{|c|}{$\begin{array}{l}\text { Married } \\
(n=82)\end{array}$} & \multicolumn{2}{|c|}{$\begin{array}{l}\text { Widow/widower } \\
\qquad(n=18)\end{array}$} & \\
\hline & & No. & $\%$ & No. & $\%$ & \\
\hline \multirow[t]{3}{*}{ Mastication } & a. Satisfactory & 49 & 56.76 & 10 & 55.60 & Total no. of patients \\
\hline & b. Partially satisfactory & 22 & 26.83 & 5 & 27.80 & Married-82,Widow/widower-18 \\
\hline & c. Not satisfactory & 11 & 13.41 & 3 & 16.70 & $\chi^{2}=0.16, p=0.92$ \\
\hline \multirow[t]{3}{*}{ Appearance } & a. Satisfactory & 25 & 30.50 & 5 & 22.80 & Total no. of patients \\
\hline & b. Partially satisfactory & 15 & 18.30 & 1 & 05.60 & Married-82,Widow/widower-18 \\
\hline & c. Not satisfactory & 42 & 51.22 & 12 & 66.70 & $\chi^{2}=2.18, p=0.34$ \\
\hline \multirow[t]{3}{*}{ Speech } & a. Satisfactory & 46 & 56.09 & 8 & 44.44 & Total no. of patients \\
\hline & b. Partially satisfactory & 17 & 20.73 & 4 & 22.22 & Married-82,Widow/widower-18 \\
\hline & c. Not satisfactory & 19 & 23.17 & 6 & 33.33 & $\chi^{2}=0.99, p=0.60$ \\
\hline \multirow[t]{3}{*}{ Comfort } & a. Satisfactory & 52 & 63.41 & 6 & 61.11 & Total no. of patients \\
\hline & b. Partially satisfactory & 23 & 28.05 & 2 & 11.11 & Married-82,Widow/widower-18 \\
\hline & c. Not satisfactory & 7 & 08.54 & 5 & 27.80 & $\chi^{2}=9.19, p=0.01$ \\
\hline \multirow[t]{3}{*}{ Health } & a. Satisfactory & 48 & 58.54 & 8 & 44.44 & Total no. of patients \\
\hline & b. Partially satisfactory & 17 & 20.73 & 6 & 33.33 & Married-82,Widow/widower-18 \\
\hline & c. Not satisfactory & 17 & 20.73 & 4 & 22.22 & $\chi^{2}=1.56, p=0.46$ \\
\hline \multirow[t]{3}{*}{ Denture care } & a. Satisfactory & 65 & 79.30 & 13 & 72.22 & Total no. of patients \\
\hline & b. Partially satisfactory & 6 & 07.32 & 1 & 05.60 & Married-82,Widow/widower-18 \\
\hline & c. Not satisfactory & 11 & 13.41 & 4 & 22.22 & $\chi^{2}=0.92, p=0.63$ \\
\hline \multirow[t]{3}{*}{ Social status } & a. Satisfactory & 41 & 50.00 & 8 & 44.44 & Total no. of patients \\
\hline & b. Partially satisfactory & 24 & 29.30 & 6 & 33.33 & Married-82,Widow/widower-18 \\
\hline & c. Not satisfactory & 17 & 20.73 & 4 & 22.22 & $\chi^{2}=0.19, p=0.91$ \\
\hline
\end{tabular}


While studying quality of satisfaction of denture treatment of illiterate group of patients with the different levels of literate group, the levels of satisfaction were found different in different literacy level. The overall level of satisfaction was found significantly higher in maximum literate level. Therefore, the level of satisfaction is higher with the increase in literacy rate. This finding revealed that literate subjects may easily understand the instruction and limitation of the artificial replacement of teeth. ${ }^{23}$ With the increase in literacy rate there is increase in treatment motivation, which may be related to awareness toward denture treatment.

While studying the compliance of subjects, toward denture in different socioeconomic status regarding motivation, the upper high income group subjects were self-motivated whereas the poor income group of subjects were motivated by others for treatment (Table 7). This finding revealed that the denture treatment is directly related to the income status of subjects. Regarding priority for denture treatment, maximum number of poor income group subjects made their denture for function followed by upper high income group subjects were found maximum in esthetics as well as function. These findings showed that poor income group subjects are more concerned to function for their denture treatment whereas the upper high and upper middle income group subjects were concerned for both esthetics and function.

The satisfaction level toward denture treatment in different economic status level revealed that in appearance and health, the upper and middle income group patients were found significantly higher in satisfaction than lower income group. Whereas in mastication, speech comfort, denture care and social status, satisfaction levels were increased with increase in socioeconomic status. This finding revealed that the levels of satisfaction are increased with increase in socioeconomic status, this may be due to either better awareness or better income status so that they can fulfill their desires. ${ }^{24}$

While considering the marital status of subjects regarding compliance toward treatment motivation, the present study has shown that there was no significant difference in married and widow/widowers. Both married and widow/widower were found equally self-motivated and also have been motivated equally by others for denture treatment (Table 9). This similarity may be due to smaller sample size in widow/widower group, which may require further research on a larger sample size in female. While considering the priority for denture treatment, the concern for esthetics was found slightly higher in married and the concern for the function was found higher in widow/ widower group. This finding revealed that the widow/widower are least concern about their esthetics rather they want to restore the function due to various psychological problems whereas the married groups were conscious for both esthetics and function, may be they were socially and professionally engaged.

While considering the satisfaction levels of different marital status group of patients toward their complete denture, it is observed that mostly the married group of patients was more satisfied in all levels of satisfaction whereas the widow/ widower group was either partially or dissatisfied. This variation in marital status regarding satisfaction level reveals that the widow/widower group of patients was psychologically depressed in all satisfaction levels.

In assessing the results of this study, several limitations are acknowledged. Despite our efforts to ensure the confidentiality of subjects' responses, some participants may have been reluctant to speak against their student dentist during the follow-up interviews, thus introducing bias. This study included small sample size and the short follow-up period. Further research efforts would include a larger, more diverse community sample.

\section{CONCLUSION}

Subject characteristics including age, gender, socioeconomic, education and marital status were associated with denture satisfaction variables but not significant with every variable. Although these variables may represent important cofactors in the patient's acceptance of dental services and may affect the way a patient perceives dental care outcomes. Aged over 65 years, male, higher income level, higher literacy and married status are significantly associated with denture satisfaction with many but not all domains.

\section{REFERENCES}

1. Steele JG, Treasure E, Pitts NB, et al. Total tooth loss in the United Kingdom in 1998 and implications for the future. $\mathrm{Br}$ Dent J 2000;189:598-603.

2. Shigli K, Hebbal M, Angadi GS. Attitudes towards replacement of teeth among patients at the institute of dental sciences. Belgaum, India. J Dent Educ 2007;71:1467-75.

3. Shigli K, Angadi GS, Hebbal M. Knowledge of prosthodontic treatment among denture-wearers and non-denture-wearers attending a dental institute in India: A survey report. Gerodontology 2007;24:211-16.

4. van Waas MA. The influence of clinical variables on patient's satisfaction with complete dentures. J Prosthet Dent 1990;63:307-10.

5. Heydecke G, Locker D, Awad MA, et al. Oral and general health-related quality of life with conventional and implant dentures. Community Dent Oral Epidemiol 2003;31:161-68.

6. Silverman WA. Doing more good than harm. Ann NY Acad Sci 1993;703:5-11.

7. Feine JS, de Grandmont P, Boudrias P, et al. Within-subject comparisons of implant-supported mandibular prostheses: Choice of prosthesis. J Dent Res 1994;73:1105-11.

8. Feine JS, Maskawi K, de Grandmont P, et al. Within-subject comparisons of implant-supported mandibular prostheses: Evaluation of masticatory function. J Dent Res 1994;73: 1646-56.

9. Awad MA, Lund JP, Shapiro SH, et al. Oral health status and treatment satisfaction with mandibular implant overdenture and conventional dentures: A randomized clinical trial in a senior population. Int J Prosthodont 2003;16:390-96.

10. van Wass MAJ. Determinants of dissatisfaction with dentures: A multiple regression analysis. J Prosthet Dent 1990;63:569-72. 
11. Vernoorn JM, Duinkerke AS, Luteijn F, et al. Assessment of denture satisfaction. Community Dent Oral Epidemiol 1988;16:364-67.

12. Ntala PC, Niarchou AP, Polyzois GL, et al. Screening of edentulous patients in a dental school population using the prosthodontic diagnostic index. Gerodontology 27:114-120.

13. Agarwal AK. Social classification: The need to update in the present scenario. Indian J Community Med 2008;33:50-51.

14. Yuuji Sato, Kazuhiro Tsuga, Yasumasa Akagawal, et al. A method for quantifying complete denture quality. J Prosthet Dent 1998;80:52-57.

15. Frauke Muller, Gerhard Wahl, Klaus Fuhr. Age-related satisfaction with complete dentures, desire for improvement and attitudes to implant treatment. Gerodontology 2006;11:7-12.

16. Mantecchini, Bassi, Pera, et al. Oral stereognosis in edentulous subjects rehabilitated with complete removable dentures. J Oral Rehabil 2002;25:185-89.

17. IIgi Baran, Gulfem Ergun, Mustafa Semiz. Sociodemographic and economic factors affecting the acceptance of removable dentures. Eur J Dent 2007;1:104-10.
18. Central Statistical Organisation (2005). Women and men in India 2004, Ministry of Statistics and Programme Implementation, CSO, Government of India, New Delhi.

19. Taylor RG. Dental survey of healthy older persons. JADA 1963;67:62-70.

20. Awad Manal A, Feine Jocelyne S. Measuring patient satisfaction with mandibular prostheses. Community Dent Oral Epidemiol 2007;26:400-05.

21. Baer ML, Elias SA, Reynolds MA. The use of psychological measures in predicting patient satisfaction with complete dentures. Int J Prosthodont 1992;5:221-26.

22. Asja Čelebić, Dubravka Knezović-Zlatarić, Milan Papić, et al. Factors related to patient satisfaction with complete denture therapy. J Gerontol A Biol Sci Med Sci 2003;58:M948-53.

23. Einar Berg, Tom Backer Johnsen, Reidun Ingebretsen. Social variables and patient acceptance of complete dentures: A study of patients attending a dental school. Acta Odontol Scand 1985;443:199-203.

24. Diehl RL, Foerster U, Sposetti VJ, et al. Factors associated with successful denture therapy. J Prosthodont 1996;5:84-90. 